\title{
ROS-Induced Oxidative Damage and Mitochondrial Dysfunction Mediated by Inhibition of SIRT3 in Cultured Cochlear Cells
}

\author{
Lingjun Zhang $\mathbb{D}$, Zhengde Du, Lu He, Wenqi Liang, Ke Liu $\mathbb{D}$, and Shusheng Gong $\mathbb{D}$ \\ Department of Otorhinolaryngology, Beijing Friendship Hospital, Capital Medical University, 95 Yongan Road, Xicheng District, \\ Beijing 100050, China
}

Correspondence should be addressed to Ke Liu; liuke@ccmu.edu.cn and Shusheng Gong; gongss1962@163.com

Received 5 February 2021; Revised 23 April 2021; Accepted 28 December 2021; Published 19 January 2022

Academic Editor: Renjie Chai

Copyright ( 2022 Lingjun Zhang et al. This is an open access article distributed under the Creative Commons Attribution License, which permits unrestricted use, distribution, and reproduction in any medium, provided the original work is properly cited.

\begin{abstract}
Sensorineural hearing loss (SNHL) is one of the most common causes of disability worldwide. Previous evidence suggests that reactive oxygen species (ROS) may play an important role in the occurrence and development of SNHL, while its mechanism remains unclear. We cultured dissected organs of Corti in medium containing different concentrations $(0,0.25,0.5,0.75,1$, and $1.25 \mathrm{mM})$ of hydrogen peroxide $\left(\mathrm{H}_{2} \mathrm{O}_{2}\right)$ and established a four-concentration model of $0,0.5,0.75$, and $1 \mathrm{mM}$ to study different degrees of damage. We examined ROS-induced mitochondrial damage and the role of sirtuin 3 (SIRT3). Our results revealed that the number of ribbon synapses and hair cells appeared significantly concentration-dependent decrease with exposure to $\mathrm{H}_{2} \mathrm{O}_{2}$. Outer hair cells (OHCs) and inner hair cells (IHCs) began to be lost, and activation of apoptosis of hair cells (HCs) was observed at $0.75 \mathrm{mM}$ and $1 \mathrm{mM} \mathrm{H}_{2} \mathrm{O}_{2}$, respectively. In contrast with the control group, the accumulation of ROS was significantly higher, and the mitochondrial membrane potential (MMP) was lower in the $\mathrm{H}_{2} \mathrm{O}_{2}$-treated groups. Furthermore, the expression of SIRT3, FOXO3A, and SOD2 proteins declined, except for an initial elevation of SIRT3 between 0 and $0.75 \mathrm{mM} \mathrm{H}_{2} \mathrm{O}_{2}$. Administration of the selective SIRT3 inhibitor 3-(1H-1,2,3-triazol-4-yl) pyridine resulted in increased damage to the cochlea, including loss of ribbon synapses and hair cells, apoptosis of hair cells, more production of ROS, and reduced mitochondrial membrane potential. Thoroughly, our results highlight that ROS-induced mitochondrial oxidative damage drives hair cell degeneration and apoptosis. Furthermore, SIRT3 is crucial for preserving mitochondrial function and protecting the cochlea from oxidative damage and may represent a possible therapeutic target for SNHL.
\end{abstract}

\section{Introduction}

As life expectancy increases, sensorineural hearing loss has become more common, affecting people's living quality [1]. There are several types of sensorineural hearing loss (SNHL), such as noised-induced hearing loss (NIHL), age-related hearing loss (ARHL), ototoxic drug-induced hearing loss (ODIHL), and inherited hearing loss. ARHL or presbycusis is a progressive decline in hearing function that is the most prevalent type of SNHL in the elderly [2-5], which is characterized by higher hearing thresholds, beginning at high frequencies and spreading toward low frequencies, accompanied by the loss of HCs and spiral ganglion neurons (SGNs) from the basal to apical turn. Noise-induced hearing loss (NIHL) is the second most prevalent type of SNHL, behind presbycusis [3]. It is typ- ically characterized by elevation in hearing thresholds and speech perception, tinnitus, and auditory processing disorders [6] due to damage to and/or death of cochlear hair cells, as well as primary auditory neurons after exposure to strong noise stimulation [7]. Platinum-based anticancer drugs and aminoglycoside antibiotics can lead to hearing loss at high frequencies and preferential damage to OHCs at the cochlea basal turn [8-10].

Reactive oxygen species (ROS) are mainly generated by the mitochondria in most mammalian cells $[11,12]$, including hydroxyl radicals and hydrogen peroxide $\left(\mathrm{H}_{2} \mathrm{O}_{2}\right)$. Growing evidence has convincingly argued that ROS and oxidative stress are responsible for the pathogenesis of various cochlear disorders, especially SNHL, including noise exposure, senility, and ototoxicity $[13,14]$. An immediate increase in ROS in the cochlea after noise exposure indicates 
that ROS are associated with early damage to cochlear hair cells and persist for 7-10 days [15]. In a model of the senescence-accelerated mouse prone 8 (SAMP8), oxidative stress and impairment in activities of antioxidant enzyme were shown to be involved in premature ARHL [16]. Moreover, ototoxic drugs can induce generation of ROS $[17,18]$. On the other hand, recent studies have suggested that before overt hearing loss happened, cochlear synaptopathy, which synapses between IHCs and cochlear afferent nerve fibers are disrupted, is more common taking place [19], although there is no direct evidence that ROS could cause loss of synapses. Rodent studies have indicated excessive release of glutamate from IHCs and insufficient energy supply can lead to cochlear synaptopathy $[20,21]$, while this all could be induced by ROS. In this study, we hoped to provide some direct evidence between ROS and cochlear synaptopathy. Much evidence implicates that mitochondrial dysfunction contributes to the occurrence and evolution of SNHL $[22,23]$. As the major inducer of ROS, it is assumed that exposure to noise, aging, and ototoxic drugs causes mitochondrial damage and, in turn, increases ROS accumulation, which eventually results in apoptosis, necrosis, and tissue damage [6]. Mitochondrial damage, lipid peroxidation, and immune inflammatory reactions are strongly associated with ROS [24-26]. ROS can also lower mitochondrial membrane potential and induce calcium overload, further damaging the mitochondria and contributing to hair cell apoptosis or necrosis [25, 27].

Given the important role ROS play in SNHL, the development and application of antioxidant drugs are becoming increasingly common. Sirtuin proteins are histone deacetylases dependent on nicotinamide adenine dinucleotide $\left(\mathrm{NAD}^{+}\right)$; they have been shown to regulate the morphology and function of mitochondria and have become the focus of research in recent years. The $\mathrm{NAD}^{+}$-dependent mitochondrial sirtuin, SIRT3, has been shown to be able to deacetylate mitochondrial respiratory chain complex subunit proteins and promote energy production [28]. SIRT3 not only increases the activity of intracellular antioxidant enzymes by activating FOXO3A but also directly deacetylates MnSOD to increase the antioxidant capacity of cells and reduce intracellular ROS levels $[29,30]$. A recent study has suggested that administration of $\mathrm{NAD}^{+}$precursor, nicotinamide riboside, can prevent spiral ganglia neurite degeneration and NIHL, which is mediated by SIRT3 [31]. In vitro, overexpression of SIRT3 can ameliorate autophagic cell death, which is dependent on superoxide anions generated from mitochondria induced by cadmium [32]. However, the direct effect of SIRT3 in cochlear basilar membranes (CBMs) has not been documented yet.

Here, we explored the hypothesis that ROS induced by exposure to different concentrations of $\mathrm{H}_{2} \mathrm{O}_{2}$ may cause mitochondrial damage and loss of ribbon synapses in vitro, which may contribute to SNHL at the cellular level. In addition, we also explored the role of SIRT3 in cochlear oxidative damage in cultured organs of Corti in vitro, and possible protective mechanisms by SIRT3 involvement were investigated.

\section{Materials and Methods}

2.1. Animals. We purchased postnatal day $3 \mathrm{C} 57 \mathrm{BL} / 6$ mice from the Experimental Animal Department of the Capital Medical University. The experimental protocols conformed to the National Institutes of Health guidelines, which were revised in 1996 (NIH Publications). Our research had got permission from the Capital Medical University Animal Ethics Committee.

2.2. Cultures. After the skin was cleaned with $75 \%$ alcohol, the postnatal day $3 \mathrm{C} 57 \mathrm{BL} / 6$ mice were euthanized by cold $\mathrm{CO}_{2}$ inhalation and decapitated painlessly. The skin was peeled off the scalp, and the skull was cut into two pieces posterior to the eye. The cochleae were removed and placed in Leibovitz's L-15 (Procell, PM151012, Wuhan, China). The organ of Corti was separated away from the stria vascularis and then adhered to separate culture plates. Each plate contained $1 \mathrm{ml} /$ well of culture medium, which comprised Dulbecco's modified Eagle's medium/Ham's F-12 (DMEM/ F-12; 1:1 Mix) (Sigma-Aldrich, D8437, St. Louis, MO, USA) with $10 \%$ bovine serum albumin (Sigma-Aldrich, A1595, St. Louis, MO, USA) and $30 \mathrm{U} / \mathrm{mL}$ penicillin G (Sigma-Aldrich, P3032, St. Louis, MO, USA). The organ of Corti explants was cultured in a humidified atmosphere with $5 \% \mathrm{CO}_{2}$ at $37^{\circ} \mathrm{C}$.

2.3. $\mathrm{H}_{2} \mathrm{O}_{2}$-Induced Oxidative Stress. According to various studies, we chose to use $\mathrm{H}_{2} \mathrm{O}_{2}$ to induce oxidative stress in vitro. First, cochlear specimens were placed in a humidified incubator for $24 \mathrm{~h}$. Then, the culture medium was discarded and substituted by fresh medium (DMEM/F-12) containing different concentrations of $\mathrm{H}_{2} \mathrm{O}_{2}(0,0.25,0.5$, $0.75,1$, and $1.25 \mathrm{mM}$ ) for $24 \mathrm{~h}$. Afterward, the medium was discarded, and the specimens were left and prepared according to subsequent studies' protocols.

Next, we evaluated the effect of SIRT3 on oxidative stress induced by $\mathrm{H}_{2} \mathrm{O}_{2}$ using 3-(1H-1,2,3-triazol-4-yl) pyridine (3-TYP) (MCE, HY-108331, Monmouth Junction, NJ, USA), an inhibitor of SIRT3. The organs of Corti were pretreated with 3-TYP $(50 \mu \mathrm{M})$ for $2 \mathrm{~h}$ prior to $\mathrm{H}_{2} \mathrm{O}_{2}$ treatment. A $100 \mathrm{mM}$ stock solution was prepared with 3-TYP dissolved in DMSO (Beyotime Biotechnology, ST038, Haimen, China) and was kept at $-80^{\circ} \mathrm{C}$ until use. We further diluted the stock solution with culture medium to appropriate concentration for administration. The highest concentration of DMSO should be under $0.1 \%$. Additionally, it has been previously shown that $50 \mu \mathrm{M} 3$-TYP does not significantly affect cell viability [32].

2.4. Immunofluorescence. The specimens were fixed with $4 \%$ paraformaldehyde for $2 \mathrm{~min}$ and then washed with PBS. Subsequently, they were incubated in $0.3 \%$ Triton X-100 containing 10\% goat serum (ZSGB-BIO, ZLI-9021, Beijing, China) at room temperature for $15 \mathrm{~min}$. Then, they were immunolabeled with monoclonal mouse anti-carboxylterminal binding protein 2 (CtBP2) (1:300, Abcam, ab204663, Cambridge, MA, USA) and rabbit anti-myosin VIIa antibody (1:300, Proteus Biosciences, 25-6790, Ramona, CA, USA) to label ribbon synapses and hair cells, 
respectively, and incubated overnight at $4^{\circ} \mathrm{C}$. The next day, the specimens were counterstained with secondary antibodies conjugated with Alexa FluorTM/ ${ }^{\otimes} 568$ and 647 (1:300, Invitrogen, A21124, A21245, Molecular Probes, Carlsbad, CA, USA) for $2 \mathrm{~h}$. The specimens were washed in PBS for $10 \mathrm{~min}$ and mounted on glass slides with $4^{\prime}, 6-$ diamidino-2-phenylindole (DAPI) (ZSGB-BIO, ZLI-9557, Beijing, China). Finally, the specimens were observed under a laser confocal microscope (Leica Microsystems, Wetzlar, Germany).

2.5. Hair Cells and Ribbon Synapse Counting. We quantified the outer hair cells (OHCs), inner hair cells (IHCs), and ribbon synapses with a $\times 63$ magnification oil-immersion objective mounted on a Leica TCS SP8 laser confocal microscope (Leica Microsystems, Wetzlar, Germany). Approximately 14-15 IHCs and 42-45 OHCs were included in each visual field. Five specimens were selected from each group to measure the average, and percentages were used to describe the survival of hair cells. We obtained the mean of ribbon synapses for each IHC using the sum of CtBP2stained puncta divided by the sum of IHC nuclei.

2.6. Determination of Mitochondria-Derived ROS. To evaluate ROS, explants were cultured in a medium containing MitoSOX (1:1000, Invitrogen, M36008, Carlsbad, CA, USA) for $30 \mathrm{~min}$ at $37^{\circ} \mathrm{C}$. Then, they were washed gently with PBS for $10 \mathrm{~min}$ and mounted on glass slides with DAPI. All steps of the procedure were protected from light. The fluorescence intensity was analyzed by laser confocal microscopy with an optimal excitation wavelength of $568 \mathrm{~nm}$ (red) and calculated by Image $J$ (National Institutes of Health, Bethesda, Maryland, USA).

2.7. Laser Confocal Microscopy. The specimens were scanned hierarchically with an interval of $0.5 \mu \mathrm{m} /$ layer by a $\times 63$ magnification oil-immersion objective mounted on a Leica TCS SP8 laser confocal microscope (Leica Microsystems, Wetzlar, Germany). The final images were superimposed. The optimal excitation wavelengths of $568 \mathrm{~nm}$ (red), $647 \mathrm{~nm}$ (far-red), and $358 \mathrm{~nm}$ (blue) for DAPI were used.

2.8. Measurement of Mitochondrial Membrane Potential. Mitochondria extraction was performed with the Tissue Mitochondria Isolation Kit (Beyotime Biotechnology, C3606, Haimen, China) based on the manufacturer's instructions as preparation. Mitochondrial membrane potential was measured using the potential-sensitive fluorescent dye JC-1 (Beyotime Biotechnology, C2006, Haimen, China). The results were detected using a multimode plate reader (EnSpireTM, Perkin Elmer Singapore Pte. Ltd. Singapore) and expressed as the ratio of the readings.

2.9. Western Blot. To determine the levels of SIRT3 and the SIRT3-related proteins, FOXO3A and SOD2, western blot was adopted. We use radioimmunoprecipitation assay lysis buffer (Beyotime Biotechnology, P0013, Haimen, China) to extract total protein and an enhanced BCA Protein Assay Kit (Beyotime Biotechnology, P0010S, Haimen, China) to quantify protein concentration. Sodium dodecyl sulfate- polyacrylamide gel electrophoresis was used to isolated protein lysates. Then, we transferred protein lysates onto polyvinylidene difluoride membranes and incubated in a $5 \%$ fat-free milk blocking solution for $1 \mathrm{~h}$. After washing in tris-buffered saline, the membranes were incubated with the primary antibodies anti-SIRT3 $(1: 1000$ CST, D22A3, Shanghai, China), anti-FOXO3A (1:1000 CST, D19A7, Shanghai, China), and anti-SOD2 (1:1000 CST, D3X8F, Shanghai, China) at $4^{\circ} \mathrm{C}$ overnight. After washing for $7 \mathrm{~min}$ three times, the membranes were subsequently incubated with the appropriate horseradish peroxidase-conjugated secondary antibody (1:5000, Santa Cruz Biotechnology, SC-2357-CM, Santa Cruz, CA, USA) at room temperature for $1 \mathrm{~h}$. Finally, the membranes were visualized using BeyoECL Plus (Beyotime Biotechnology, P0018S, Haimen, China). We used the Image-Pro Plus 6.0 software (Media Cybernetics, Rockville, MD, USA) to analyze quantification of these proteins.

2.10. Apoptosis Staining. We used a terminal deoxynucleotidyl transferase-mediated deoxy uridine triphosphate nickend labeling staining kit TUNEL POD (Roche Molecular Biochemicals, 11772465001, Mannheim, Germany) to detect hair cell apoptosis. After being incubated in $0.3 \%$ Triton X100 containing $10 \%$ goat serum (ZSGB-BIO, ZLI-9021, Beijing, China) at room temperature for $15 \mathrm{~min}$, the specimens were immunolabeled with rabbit anti-myosin VIIa antibody (1:300, Proteus Biosciences, 25-6790, Ramona, CA, USA) and incubated overnight at $4^{\circ} \mathrm{C}$. The next day, the specimens were first counterstained with secondary antibodies conjugated with Alexa FluorTM/ ${ }^{\circledR} 647$ (1:300, Invitrogen, A21124, A21245, Molecular Probes, Carlsbad, CA, USA) for $2 \mathrm{~h}$. And then, the label reaction with TUNEL POD was carried out for $1 \mathrm{~h}$ at $37^{\circ} \mathrm{C}$. Wash the specimens with PBS for $10 \mathrm{~min}$ and mount them on glass slides with DAPI (ZSGB-BIO, ZLI-9557, Beijing, China). Finally, the specimens were observed under a laser confocal microscope (Leica Microsystems, Wetzlar, Germany).

2.11. Statistical Analyses. We use GraphPad Prism 8 (GraphPad Software, Inc., La Jolla, CA, USA) to perform statistical cartography and illustrate our data with mean \pm standard error. The paired-sample $t$-test was performed to evaluate differences between the different groups. Statistical significance was set at $P<0.05$.

\section{Results}

3.1. $\mathrm{H}_{2} \mathrm{O}_{2}$ Induces Dose-Dependent Ribbon Synapse, and Hair Cells Decline: Model Building. First, we determined the changes in the number of ribbon synapses and the survival of hair cells under different $\mathrm{H}_{2} \mathrm{O}_{2}$ concentrations. Our findings showed that after a $24 \mathrm{~h}$ exposure to $\mathrm{H}_{2} \mathrm{O}_{2}$, the number of ribbon synapses and cochlear hair cells was significantly decreased in a concentration-dependent manner. We recorded the concentrations at which there was apparent loss of ribbon synapses with no loss of hair cells, apparent loss of ribbon synapses and $\mathrm{OHCs}$, and apparent loss of IHCs (Figure 1(a)). 

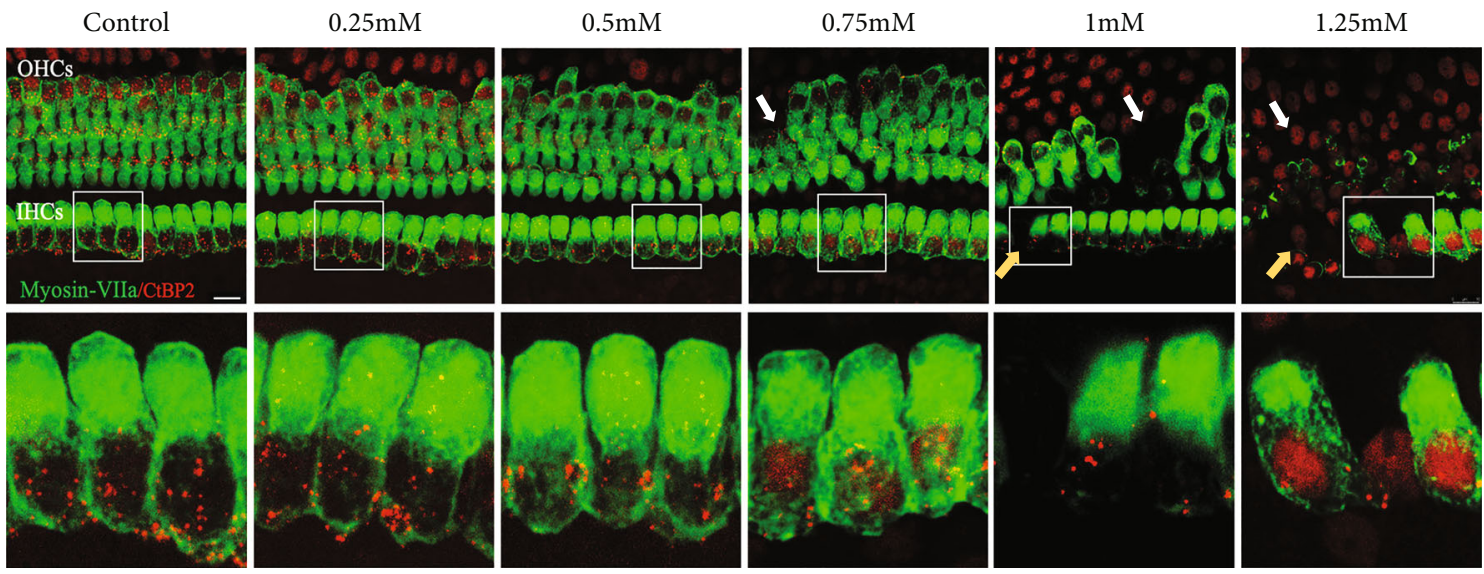

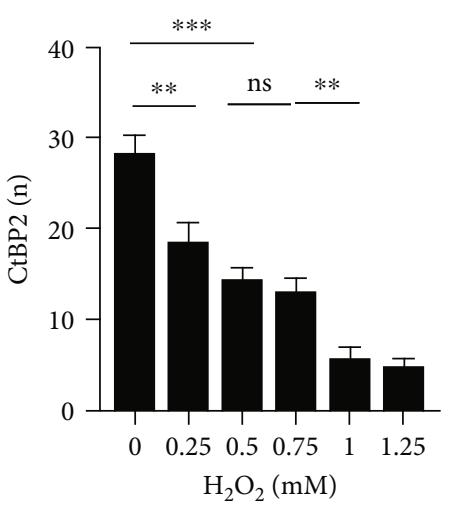

(b)

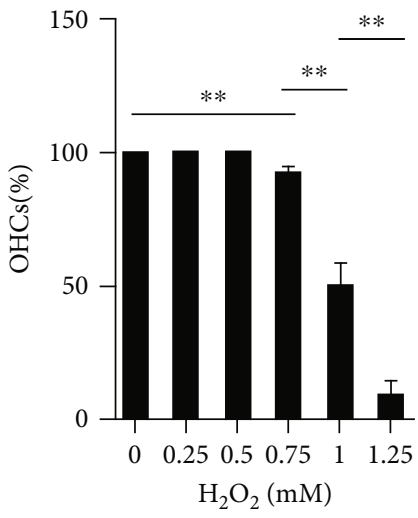

(c)

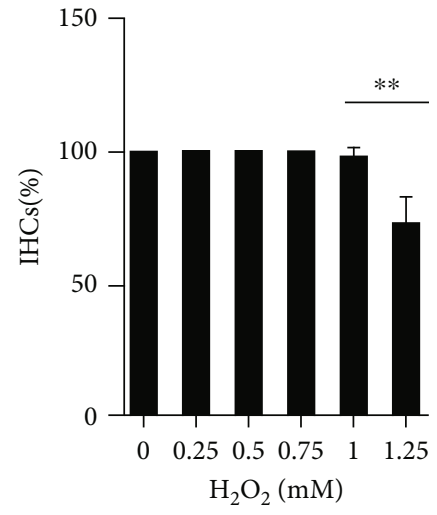

(d)

Figure 1: $\mathrm{H}_{2} \mathrm{O}_{2}$ induces dose-dependent loss of ribbon synapses and hair cells at the $0,0.25,0.50 .75,1$, and $1.25 \mathrm{mM}$ concentrations. (a) Confocal images showing ribbon synapses (red) and hair cells (green) in the middle region of cochlear explants at each concentration. The white arrows indicate OHC damaged areas. Yellow arrows indicate IHC damaged areas. (b) Quantification of ribbon synapses at each concentration. (c) Rate of OHC survival at each concentration. (d) Rate of IHC survival at each concentration. Data are expressed as means $\pm \mathrm{SD}\left(n=5\right.$ organ of Corti per concentration). ${ }^{*} P<0.05,{ }^{* *} P<0.01$, and ${ }^{* * *} P<0.001$. Scale bar $=10 \mu \mathrm{m}$.

The number of ribbon synapses in the middle turn of the organ of Corti in the control, 0.25, 0.5, 0.75, 1, and $1.25 \mathrm{mM}$ groups was $28.2 \pm 81.99,18.5 \pm 2.11,14.36 \pm$ $1.37,12.96 \pm 1.63,5.62 \pm 1.36$, and $4.75 \pm 0.92$, respectively (Figure 1(b)). In contrast with the $0.25 \mathrm{mM}$ group, there were significantly fewer ribbon synapses in the $0.5 \mathrm{mM}$ group in comparison with the control group $(P<0.001)$. There was no significant difference in the number of ribbon synapses in the $0.5 \mathrm{mM}$ and $0.75 \mathrm{mM}$ groups $(P>0.05)$, while the number of ribbon synapses in the $1 \mathrm{mM}$ group was efficaciously lower than that in the $0.75 \mathrm{mM}$ group $(P<0.01)$. And no significant difference was found in the number of ribbon synapses between the $1 \mathrm{mM}$ and $1.25 \mathrm{mM}$ groups.

Regarding the survival of hair cells, we investigated the whole cochlear basilar membranes (Figures 1(c) and 1(d)). In the $0.25 \mathrm{mM}$ and $0.5 \mathrm{mM}$ groups, hair cells remained intact, and there were no obvious alterations in their quantities, although the arrangement of hair cells in the apex of the cochlea was disordered in the $0.5 \mathrm{mM}$ group. At a concentration of $0.75 \mathrm{mM} \mathrm{H}_{2} \mathrm{O}_{2}, 92.75 \pm 1.53 \%$ of OHCs $(P<0.01)$ and all IHCs remained. In the $1 \mathrm{mM}$ group, approximately $50 \%$ of OHCs survived $(50.10 \pm 7.99 \%)$, which was significantly different compared with the $0.75 \mathrm{mM}$ group $(P<$ 0.01 ), and $97.92 \pm 2.95 \%$ of IHC survived. There was extensive hair cell loss in the $1.25 \mathrm{mM}$ group; only $9.13 \pm 5.54 \%$ and $72.35 \pm 9.79 \%$ of OHCs and IHCs survived, respectively, which was significantly different from the $1 \mathrm{mM}$ group (both $P<0.01)$. According to the results above, we built a model of four concentrations: $0,0.5,0.75$, and $1 \mathrm{mM}$ for the following study.

3.2. Pharmacological Suppression of SIRT3 and Aggravated Oxidative Damage to the Cochlea. To investigate the impact of SIRT3 mitigation on $\mathrm{H}_{2} \mathrm{O}_{2}$-induced oxidative damage, we used a selective SIRT3 inhibitor, 3-TYP [33]. Previous research by Pi et al. revealed that 3-TYP can inhibit SIRT3 activity but does not downregulate protein expression [32].

We first quantified the number of ribbon synapses and analyzed the survival of hair cells in the groups established above. Our results revealed that the damage to ribbon synapses and hair cells was dependent on the concentration of 

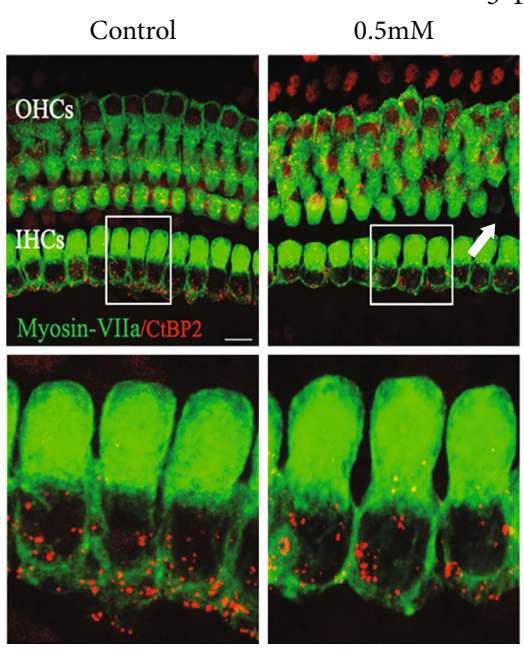

3-TYP

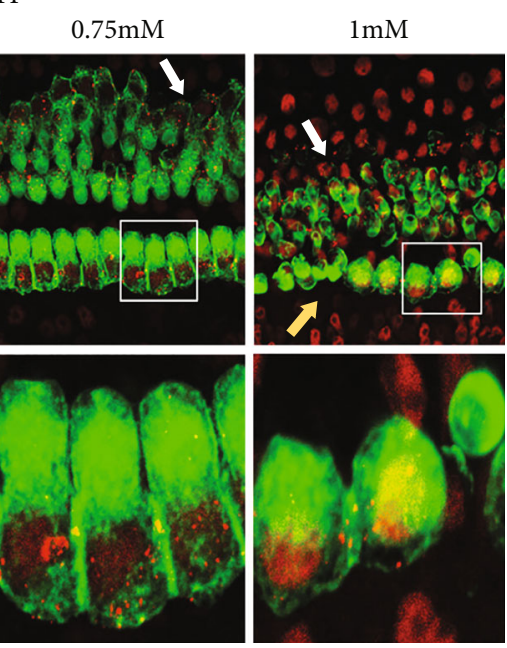

(a)

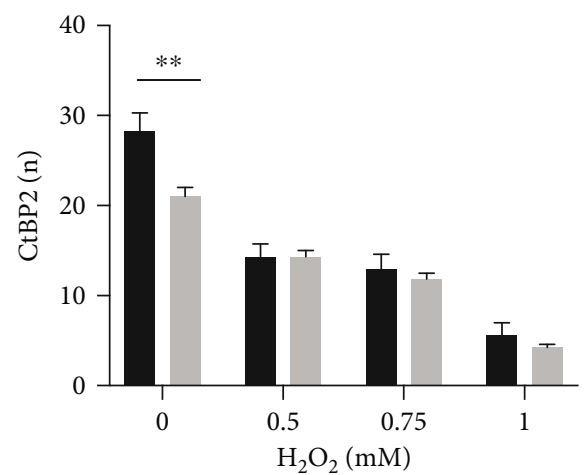

(b)

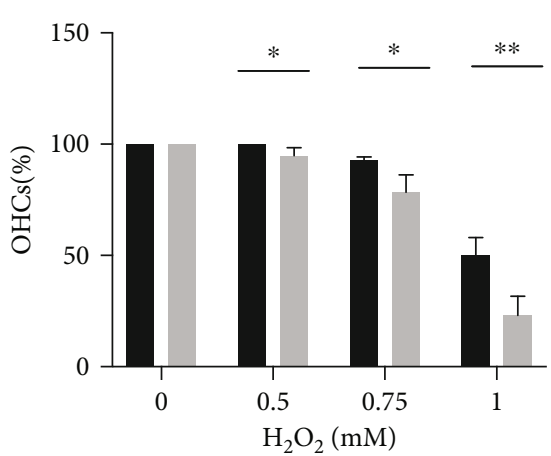

(c)

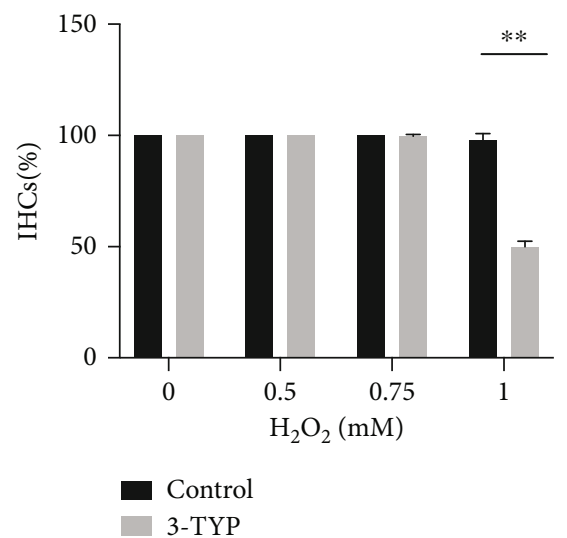

(d)

Figure 2: $\mathrm{H}_{2} \mathrm{O}_{2}$ induces aggravated oxidative damage to cochlear by suppression of SIRT3. (a) Representative images of ribbon synapses (red) and HCs (green) in the middle region of cochlear explants in 0, 0.5, 0.75, and $1 \mathrm{mM}$ groups pretreated with 3-TYP. The white arrows indicate OHC damaged areas. Yellow arrows indicate IHC damaged areas. (b) Comparison of ribbon synapse at each concentration between matched and 3-TYP groups. (c) Comparison of OHC survival at each concentration between matched and 3-TYP groups. (d) Comparison of IHC survival at each concentration between matched and 3-TYP groups. Data are shown as means \pm SD ( $n=5$ in each group). ${ }^{*} P<0.05,{ }^{* *} P<0.01$, and ${ }^{* * *} P<0.001$. Scale bar $=10 \mu \mathrm{m}$.

$\mathrm{H}_{2} \mathrm{O}_{2}$ (Figure 2). In the presence of 3-TYP, the number of ribbon synapses in the middle turn of the organ of Corti in the control, 0.5, 0.75, and $1 \mathrm{mM}$ groups declined with $21.03 \pm 0.98,14.31 \pm 0.72,11.85 \pm 0.62$, and $4.25 \pm 0.33$, respectively (Figure $2(\mathrm{~b})$ ). Interestingly, after pretreated with 3-TYP, no significant difference was discovered in the number of ribbon synapses between these $\mathrm{H}_{2} \mathrm{O}_{2}$ concentrations, except in the control group, in which the number was declined $(P<0.01)$ (Figure $2(\mathrm{~b}))$.

However, after pretreated with 3-TYP, HCs in $\mathrm{H}_{2} \mathrm{O}_{2}$ groups appeared more disorganized and morphologically abnormal, as compared Figure 1(a) with Figure 2(a). Furthermore, as shown in Figure 2(c), there was a significantly lower rate of $\mathrm{OHC}$ survival in the $0.5 \mathrm{mM}$ group compared with the group that was not treated with 3 -TYP $(P<0.05)$. A similar tendency also appeared in the $0.75 \mathrm{mM}$ and $1 \mathrm{mM}$ groups $(P<0.05$ and $P<0.01$, respectively). Regarding the survival rate of IHCs (Figure 2(d)), in the $0.75 \mathrm{mM}$ group, we observed that IHC loss began, and those that remained were chaotically arranged. In the $1 \mathrm{mM}$ group treated with 3-TYP, only $49.91 \pm 2.47 \%$ IHCs survived, compared with $97.92 \pm 2.95 \%$ in the $1 \mathrm{mM}$ group without 3 -TYP $(P<0.01)$. However, there was no significant difference between the rate of IHC survival in both control and $0.5 \mathrm{mM}$ groups. These results revealed that 3-TYP could inhibit intracellular antioxidant activity and aggravate oxidative stress, increasing the damage to the ribbon synapse and hair cells.

3.3. $\mathrm{H}_{2} \mathrm{O}_{2}$ Induces Increased Mitochondrial ROS in CBMs, and This Is Enhanced with 3-TYP Treatment. We measured the levels of mitochondrial ROS using MitoSOX staining. The red fluorescence intensity of MitoSOX staining in the groups treated with $\mathrm{H}_{2} \mathrm{O}_{2}$ was significantly more apparent than that in the control group, and the level was largely 


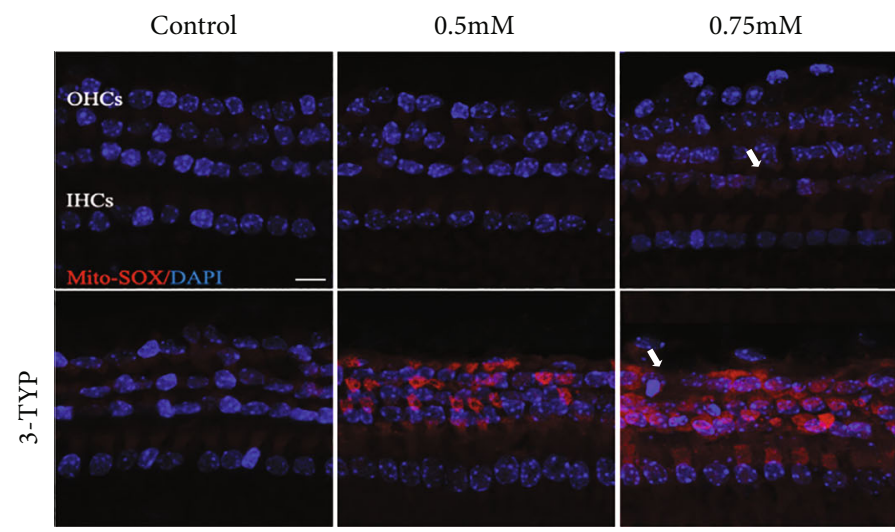

(a)

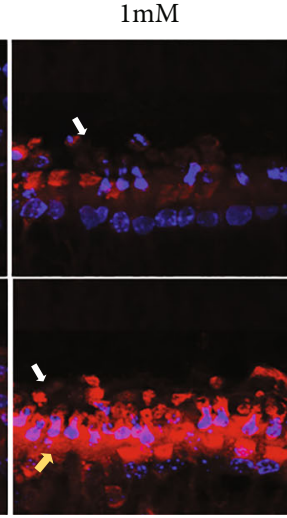
FIgURE 3: $\mathrm{H}_{2} \mathrm{O}_{2}$ induces ROS generation, which is enhanced by 3 -TYP. (a) Confocal images of MitoSOX staining (red) in the $0,0.5,0.75$,
and $1 \mathrm{mM}$ groups pretreated with or without 3 -TYP. The white arrows indicate OHC damaged areas. Yellow arrows indicate IHC damaged areas. (b) Relative level of fluorescence intensity in each group. Data are expressed as means \pm SD ( $n=5$ organ of Corti per concentration). ${ }^{*} P<0.05,{ }^{* *} P<0.01$, and ${ }^{* * *} P<0.001$. Scale bar $=10 \mu \mathrm{m}$.

dependent on the concentrations of $\mathrm{H}_{2} \mathrm{O}_{2}$, with the highest in the $1 \mathrm{mM}$ group, regardless of pretreatment with 3-TYP, as shown in Figure 3. The quantitative comparison was performed using red fluorescence intensity (Figure 3(b)). Relative staining in the $0.5,0.75$, and $1 \mathrm{mM}$ groups rose by $1.07 \pm 0.10$ - fold, $1.42 \pm 0.15$ - fold, and $2.27 \pm 0.37$ - fold, respectively. No significant difference was shown in the relative ROS levels between the 0 and $0.5 \mathrm{mM}$ groups. Relative fluorescence intensity in the $0.5 \mathrm{mM}$ group was significantly lower than that in the $0.75 \mathrm{mM}$ group $(P<0.05)$, and relative fluorescence intensity in the $0.75 \mathrm{mM}$ group was significantly lower than that in the $1 \mathrm{mM}$ group $(P<0.05)$.

After pre-treated with 3-TYP, the relative fluorescence intensity was increased in each concentration group $(0,0.5$, 0.75 , and $1 \mathrm{mM}$ ), by $1.33 \pm 0.15$ - fold, $1.96 \pm 0.19-$ fold, $2.43 \pm 0.18$ - fold, and $2.74 \pm 0.16$, respectively. Relative staining was significantly higher as $\mathrm{H}_{2} \mathrm{O}_{2}$ concentrations higher: control vs. $0.5 \mathrm{mM}, P<0.01,0.5$ vs. $0.75 \mathrm{mM}, P<$ 0.05 , and 0.75 vs. $1 \mathrm{mM}, P<0.05$, respectively. Furthermore, the levels of relative fluorescence intensity in control, 0.5 , and $0.75 \mathrm{mM} 3$-TYP pretreated groups were significantly higher than in the nontreated matched group with $P<0.01$. Thus, it indicated that mitochondrial ROS levels increased in response to $\mathrm{H}_{2} \mathrm{O}_{2}$ treatment in vitro. Furthermore, this may be enhanced by inhibition of SIRT3.

3.4. $\mathrm{H}_{2} \mathrm{O}_{2}$ Induces Mitochondrial Dysfunction in CBMs, Which Is Intensified by 3-TYP Treatment. The MMP was used to evaluate mitochondrial function. As shown in Figure 4, MMP levels in the $0,0.5,0.75$, and $1 \mathrm{mM}$ groups were $2.23 \pm 0.27,1.76 \pm 0.09,1.67 \pm 0.12$, and $1.43 \pm 0.10$, respectively. Our results showed that mitochondrial function was inversely related to $\mathrm{H}_{2} \mathrm{O}_{2}$ concentration. The MMP level in the $0.5 \mathrm{mM}$ and $0.75 \mathrm{mM}$ groups was not significantly different. The MMP level in the $0.5 \mathrm{mM}$ group was significantly lower than that in the control group $(P<0.01)$, as was the MMP level in the $1 \mathrm{mM}$ group compared to that in the $0.75 \mathrm{mM}$ group $(P<0.01)$. The result also illustrated the

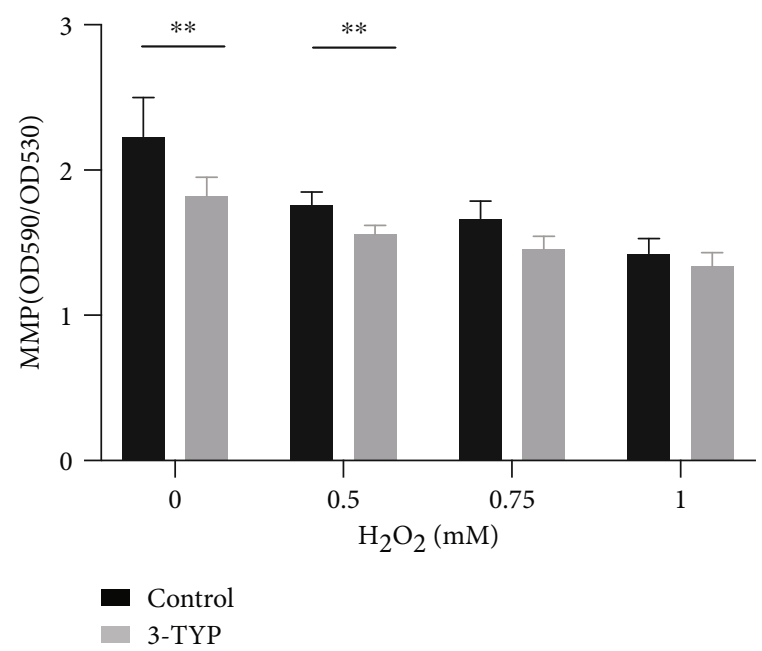

FIgURE 4: Comparison of MMP levels in the 0, 0.5, 0.75, and $1 \mathrm{mM}$ groups between matched nontreated and 3-TYP-treated groups. Data are expressed as means $\pm \mathrm{SD}(n=5$ organ of Corti per concentration). ${ }^{*} P<0.05,{ }^{* *} P<0.01$, and ${ }^{* * *} P<0.001$.

MMP levels in the same concentration groups pretreated with 3-TYP; these were $1.82 \pm 0.13,1.56 \pm 0.06,1.46 \pm 0.08$, and $1.34 \pm 0.10$, respectively, which also demonstrated a concentration-dependent decline. There was a significant difference in MMP between the control and $0.5 \mathrm{mM}$ groups $(P<0.01)$ and the $0.5 \mathrm{mM}$ and $1 \mathrm{mM}$ groups $(P<0.05)$. To investigate the effect of SIRT3 suppression, we compared the MMP level between the 3-TYP pretreated group and nontreated group. The MMP level in each 3-TYP pretreated group was lower than in the nontreated matched group, with a significant difference in the 0 and $0.5 \mathrm{mM}$ groups $(P<0.01)$. Our findings indicated that $\mathrm{H}_{2} \mathrm{O}_{2}$ resulted in a concentrationdependent decrease in mitochondrial membrane potential, and SIRT3 played an important role in protecting mitochondrial function. 


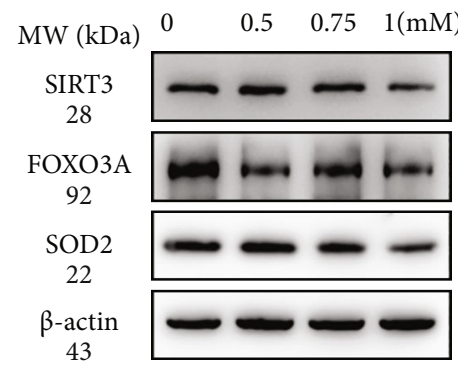

(a)

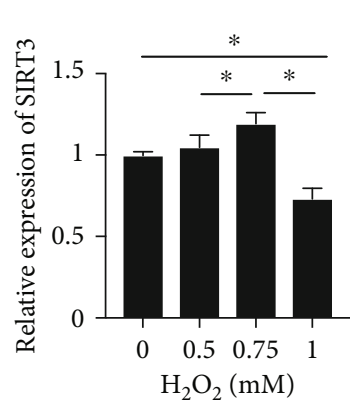

(b)

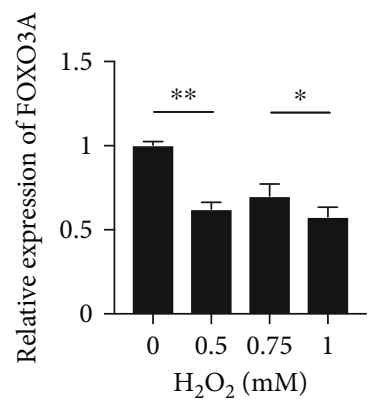

(c)

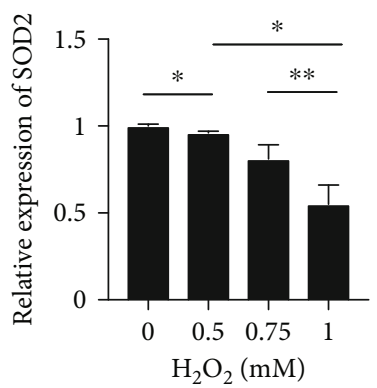

(d)

FIGURE 5: $\mathrm{H}_{2} \mathrm{O}_{2}$ induces downregulation of SIRT3, FOXO3A, and SOD2. (a) Representative western blot analysis using antibodies against SIRT3, FOXO3A, SOD2, and $\beta$-actin in CBMs. (b) Protein expression of SIRT3 in the $0,0.5,0.75$, and 1 mM groups. (c) Protein expression of FOXO3A in the 0, 0.5, 0.75, and $1 \mathrm{mM}$ groups. (d) Protein expression of SOD2 in the 0, 0.5, 0.75, and $1 \mathrm{mM}$ groups. Data are expressed as means $\pm \mathrm{SD}\left(n=5\right.$ organ of Corti per concentration). ${ }^{*} P<0.05,{ }^{* *} P<0.01$, and ${ }^{* * *} P<0.001$.

3.5. $\mathrm{H}_{2} \mathrm{O}_{2}$ Downregulates SIRT3 and SIRT3-Dependent Protein Expression. As previously mentioned, SIRT3 is crucial in oxidation resistance and protects cells from oxidative damage. We analyzed the expression of SIRT3 and the related proteins FOXO3A and SOD2 by western blotting to confirm this effect (Figure 5). As shown in Figure 5(b), the level of SIRT3 protein was significantly higher in the $0.75 \mathrm{mM}$ group than in the $0.5 \mathrm{mM}$ and control groups $(1.19 \pm 0.07-$ fold vs. $1.05 \pm 0.08-$ fold, $P<0.05 ; 1.19 \pm$ 0.07 - fold vs. $1.00 \pm 0.02-$ fold, $P<0.05$, respectively). However, in the $1 \mathrm{mM}$ group, the protein level was dramatically decreased to $0.73 \pm 0.07$ - fold, which was significantly lower than in any other group $(P<0.05)$. This suggested that stimulation by $\mathrm{H}_{2} \mathrm{O}_{2}$ initially elevated the level of SIRT3 protein, which could help cells to resist oxidative damage to a certain degree. However, this elevation was limited and finally declined. The level of FOXO3A protein, a downstream target of SIRT3, generally declined (Figure 5(c)). By contrast with the control group, the level of FOXO3A was decreased in the $0.5 \mathrm{mM}, 0.75 \mathrm{mM}$, and $1 \mathrm{mM}$ groups by $0.62 \pm 0.04-$ fold $(P<0.01), 0.70 \pm 0.07-$ fold $(P<0.05)$, and $0.58 \pm 0.06-$ fold $(P<0.01)$, respectively. Compared with the control group, the expression of SOD2 protein declined stably to $0.96 \pm 0.01$ - fold in the $0.5 \mathrm{mM}$ group $(P<0.05), 0.81 \pm 0.08-$ fold in the $0.75 \mathrm{mM}$ group $(P<$ $0.05)$, and $0.55 \pm 0.11$ - fold in the $1 \mathrm{mM}$ group $(P<0.05)$. Moreover, the levels were significantly different between the $0.5 \mathrm{mM}$ and $1 \mathrm{mM}$ groups and the $0.75 \mathrm{mM}$ and $1 \mathrm{mM}$ groups, although no difference was noticed in expression levels between the $0.5 \mathrm{mM}$ and $0.75 \mathrm{mM}$ groups, which may be related to statistical error. The decrease in the expression of these two downstream proteins indicates that despite elevated SIRT3 expression, $\mathrm{H}_{2} \mathrm{O}_{2}$ activated the oxidative reaction and degraded the expression of antioxidant proteins, causing damage to tissues. Together, these results provide evidence that $\mathrm{H}_{2} \mathrm{O}_{2}$ could induce the downregulation of SIRT3 and SIRT3-related proteins, thus inhibiting the antioxidant reaction and causing damage.

3.6. Hair Cell Apoptosis Induced by $\mathrm{H}_{2} \mathrm{O}_{2}$ and Intensified by 3-TYP Treatment. As mentioned above, the loss of hair cells appeared significantly $\mathrm{H}_{2} \mathrm{O}_{2}$ concentration-dependent, which may due to the activation of apoptosis induced by $\mathrm{H}_{2} \mathrm{O}_{2}$. To confirm the occurrence of apoptosis, we used TUNEL POD staining. As shown in Figure 6, the TUNELpositive cells were first observed in the $\mathrm{OHC}$ area in the $0.75 \mathrm{mM}$ group. In the $1 \mathrm{mM}$ group, a large number of $\mathrm{OHCs}$ appeared apoptosis and a portion of IHCs lost directly. After treatment with 3-TYP, apoptosis of hair cells occurred earlier and severer, as the TUNEL-positive cells were first observed in the OHC area in the $0.5 \mathrm{mM}$ group and more TUNELpositive OHCs were in the $0.75 \mathrm{mM}$ group. Furthermore, in the $1 \mathrm{mM}$ group, none of OHCs survived and almost half of IHCs lost directly. However, we still could observe a TUNEL-positive cell in IHC area. This suggests that $\mathrm{H}_{2} \mathrm{O}_{2}$ induces apoptosis of hair cells in the CBMs, and administration of 3-TYP could exacerbate the occurrence of apoptosis.

\section{Discussion}

Hair cells mainly function in turning the sound wave energy into electric signals [34]. Although SNHL could be caused by 

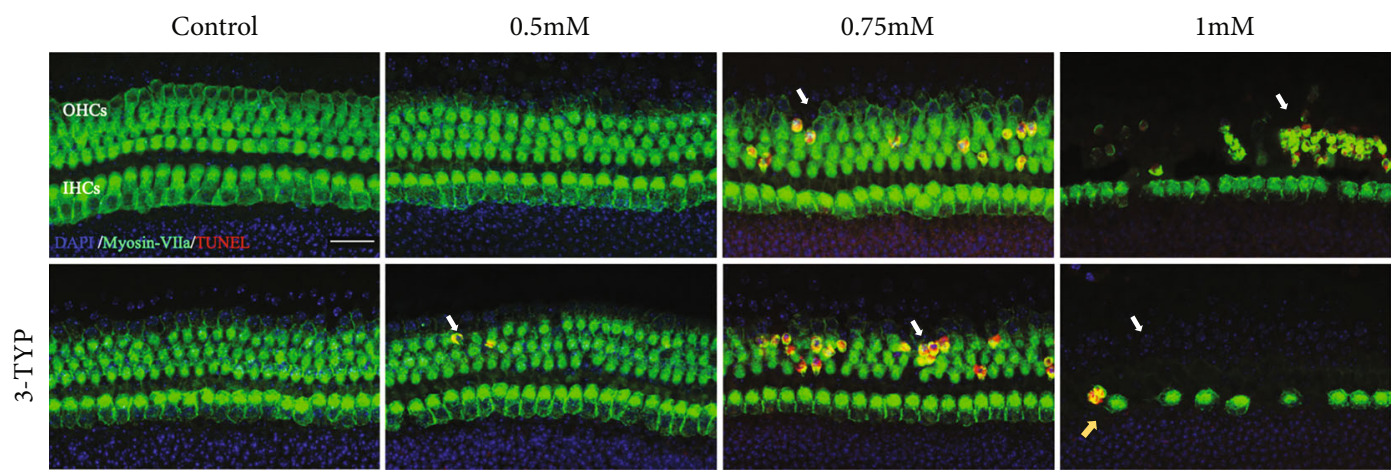

FIGURE 6: $\mathrm{H}_{2} \mathrm{O}_{2}$ induce occurrence of hair cell apoptosis, and this is intensified by 3-TYP. Representative images of TUNEL staining (red) and hair cells (green) in $0,0.5,0.75$, and $1 \mathrm{mM}$ groups pretreated with or without 3-TYP. The white arrows indicate TUNEL-positive cells in OHC areas. Yellow arrows indicate TUNEL-positive cells in IHC areas. Scale bar $=25 \mu \mathrm{m}$.

many factors, including genetic factors, aging, chronic cochlear infections, infectious diseases, ototoxic drugs, and noise exposure, most of the SNHL occurs due to the irreversible loss of HCs and spiral ganglion neurons (SGNs), both of which have limited regeneration ability in adult mammals [35]. Thus, how to protect the cochlear HC is a key scientific question in the hearing research field. As the human cochlear is inaccessible, in vitro animal models could be a crucial way to study SNHL. Here, we studied the oxidative damage to the organ of Corti caused by $\mathrm{H}_{2} \mathrm{O}_{2}$ and further investigated the changes of the oxidative damage after inhibition of SIRT3. Accumulation of ROS could result in various degrees of loss of hair cells and ribbon synapses, decrease of mitochondrial membrane potential, and decrease of SIRT3 and related protein expression. Furthermore, under the administration of 3-TYP, oxidative damage was aggravated.

4.1. Apoptosis May Contribute to Loss of HCs. It has been proven that hearing mostly depends on hair cells to convert mechanical stimulation to electrochemical activity and the IHC ribbon synapses to transmit electrochemical signals $[36,37]$. OHCs are mainly responsible for the active mechanical amplification process, which facilitates high sensitivity and good frequency resolution. IHCs are responsible for sound detection and provide afferent auditory neurotransmission to the brain $[11,38,39]$. In this study, cochlear sensory hair cells and IHC ribbon synapses in cochlear explants exhibited dose-dependent cytotoxic effects when exposed to $\mathrm{H}_{2} \mathrm{O}_{2}$. At certain concentration group, we viewed an enormous loss of OHCs and a slight loss of IHCs. Previous research has shown that the degeneration and loss of hair cells are largely due to the activation of apoptosis [40, 41]. Apoptosis occurs in two ways: the extrinsic pathway, activated by death receptors, and the intrinsic pathway, initiated by a change in mitochondrial membrane permeability [42-44]. Mitochondrial dysfunction causes the permeability transition pore to open in an irreparable way, which subsequently leads to rise of the permeability of mitochondrial outer membrane $[42,45]$. Moreover, the accumulation of ROS, decrease of the mitochondrial membrane potential, and release of apoptosis-related factors and cytochrome $c$ were induced by mitochondrial dysfunction spark apoptotic and necrotic cell death pathways as well $[44,46]$.

4.2. Lack of Energy May Contribute to Synaptic Loss. Our results indicate that the intrinsic pathway of apoptosis due to mitochondrial dysfunction and overgeneration of ROS may contribute to the loss of hair cells in the cochlea. Our results also showed that exposure to $\mathrm{H}_{2} \mathrm{O}_{2}$ significantly decreased the number of ribbon synapses, even at low concentrations. The concomitant decline in the mitochondrial membrane potential decreases adenosine triphosphate (ATP) levels in the cochlea, which becomes insufficient for the transportation of ribbon synaptic vesicles and maintenance of ribbon synapses' function [41]. Considering oxidative stress, related studies have shown that inhibition of AMPK, a key cellular energy sensor related to ATP production, could attenuate the loss of outer hair cells and ribbon synapses and preserved auditory function after noise exposure [47]. Recent animal studies have shown that synaptic loss can occur without permanent hearing threshold shifts after noise exposure $[48,49]$, suggesting that synaptopathy is a significant marker of early noise-induced hearing loss.

\subsection{Accumulation of ROS Reduces MMP and Triggers} Oxidative Damage. To evaluate the level of ROS, we used MitoSOX staining, and our results demonstrated that the higher the concentration, the greater the accumulation of ROS and the more severe the damage to the cochlea. Most mammalian cells generate ROS, which are regarded as toxic products of cellular metabolism by the mitochondria, and can act as signalling molecules to regulate various physiological processes [50]. It is assumed that noise exposure induces mitochondrial damage and, in turn, increases ROS accumulation. A considerable amount of ROS can trigger progressive oxidative damage, promote oxidation of the mitochondrial DNA and proteins [51], and induce lipid peroxidation products, which can result in apoptosis and reduce cochlear blood flow [6]. Normal mitochondrial membrane potential is a prerequisite for maintaining oxidative phosphorylation of mitochondria and ATP production. The cumulative burden of ROS production can lead to MMP breakdown, which further results in energy deficiency and mitochondrial dysfunction $[41,52]$. In this study, the 
MMP levels in both groups (with and without 3-TYP) decreased as $\mathrm{H}_{2} \mathrm{O}_{2}$ concentration increased. In particular, the pharmacological inhibitor 3-TYP of SIRT3 intensified the decline of MMP.

4.4. Potential Role of SIRT3 in Hearing Protection and Mitohormesis. SIRT3 is the major mitochondrial deacetylase and is expressed in metabolically active tissues such as the liver, kidney, and heart [28]. It appears that it can resist various mitochondrial stresses, especially by utilizing cellular antioxidant systems to combat oxidative stress. FOXO3A is a forkhead transcription factor that is deacetylated by SIRT3 to increase the transcription of key antioxidant genes, including SOD2 and catalase, and protects mitochondria from further oxidative stress $[53,54]$. SOD2 is responsible for reducing ROS and protecting against oxidative stress and is also activated by SIRT3-mediated deacetylation [30]. Additionally, SIRT3 directly deacetylates mitochondrial isocitrate dehydrogenase, IDH2, and stimulates its activity [55], which helps to maintain the mitochondrial pool of NADPH for conversion of reduced glutathione [56-58].

To detect the expression of SIRT3, FOXO3A, and SOD2 proteins, we performed western blot analysis. Our results revealed that under moderate amounts of ROS (i.e., the $0.5 \mathrm{mM}$ and $0.75 \mathrm{mM} \mathrm{H}_{2} \mathrm{O}_{2}$ groups), the level of SIRT3 initially increased and subsequently decreased in the $1 \mathrm{mM}$ group. We suggest that this may correspond with mitohormesis, which means that the effects caused by a stressor may be beneficial when its level is relatively low and deleterious when it is high [59]. It has been reported that the level of SIRT3 increases under calorie restriction (CR), fasting, and exercise training in different tissues [55, 60]. Under calorie restriction, a moderate increase in ROS can trigger oxidative stress resistance activities to attenuate oxidative cellular damage $[59,61]$. However, large or chronic increases in ROS may result in damage or cell death, as they exceed the capacity to maintaining homeostasis; thus, the balance is broken. It is noteworthy that oxidative stress is commonly associated with mitochondrial hyperacetylation $[60,62]$. We assume that the activities of antioxidants and related enzymes such as FOXO3A and SOD2 are dampened through direct acetylation $[58,63,64]$. In this study, we found that the levels of FOXO3A and SOD2 decreased as the $\mathrm{H}_{2} \mathrm{O}_{2}$ concentration increased. As mentioned above, a moderate ROS rise increases SIRT3 transcription, thus helping to achieve a new hormetic steady state. The increased levels of SIRT3 can then deacetylate FOXO3A and SOD2 to defend against oxidative stress, but this capacity is limited. Mitochondrial hyperacetylation fails in the function of antioxidant enzymes and excess neutralization with oxidation products and ultimately reduces the expression of SIRT3, FOXO3A, and SOD2 and worsens oxidative stress. Interestingly, the expression of FOXO3A protein rose slightly in the $0.75 \mathrm{mM} \mathrm{H}_{2} \mathrm{O}_{2}$ group, which may probably due to dramatical expression of its upstream protein SIRT3, as assumed. Also, statistical bias cannot be ruled out, either.

To provide direct evidence that SIRT3 is a key factor in the preservation of mitochondrial function and antioxidation, we performed biochemical experiments with the selec- tive SIRT3 inhibitor, 3-TYP. 3-TYP inhibits SIRT3 activity but does not affect SIRT3 protein expression [32, 33]. Our findings illustrated that inhibition of SIRT3 exacerbated the level of ROS, loss of hair cells and ribbon synapses, and decreased MMP. A previous study in cultured HepG2 cells had suggested that activation of SIRT3 could suppress mitochondrial-derived ROS-stimulated autophagic cell death induced by cadmium through SIRT3/SOD2 pathway [32]. ROS is important for osteoclasts in differentiation and activation induced by receptor activator of NF- $\kappa \mathrm{B}$ ligand (RANKL). Suppression of SOD2 activity by SIRT3targeted siRNA could increase ROS levels and raise osteoclastogenesis [65]. High expression of $\mathrm{xCT}$, which is the member 11 of solute carrier family 7 (SLC7A11), was commonly along with increased levels of ROS and sensitivity to glucose deprivation in breast cancer cells. Downregulation of SIRT3 further increased the levels of ROS and promoted xCT-related cell death [66]. Adjudin, a lonidamine analogue, could protect cochlear HCs from gentamicin-induced damage mediated by the SIRT3-ROS axis in vitro [67]. Furthermore, an in vivo study revealed that SIRT3 ${ }^{-1-}$ mice were more susceptible to NIHL, and SIRT3 ${ }^{-1-}$ mice treated with nicotinamide riboside exhibited less protection from both TTS and PTS after noise exposure [31]. On the other hand, it is interesting that significant reduction in the number of ribbon synapses was only discovered in the control group pretreated with 3TYP. As mentioned above, great evidence shows that the level of SIRT3 increases under stress like CR. We have reason to believe that the protective effect for ribbon synapses of higher expression of SIRT3 induced by $\mathrm{H}_{2} \mathrm{O}_{2}$ is stronger than inhibition of activity, while in the control group, only the activity of SIRT3 was inhibited, and the loss of ribbon synapses became severer.

However, in vitro research provides the suggestion for studies in vivo but is different from in vivo research because of the complex internal environment and variable influencing factors. Next, we hope to further study the mitochondrial oxidative damage and deacetylation of SIRT3 in NIHL in vivo.

\section{Conclusion}

Overall, we propose that ROS and oxidative stress are major causes of SNHL. Furthermore, SIRT3 is crucial for preserving mitochondrial function and protecting the cochlea from oxidative damage. Additionally, we imitate different phases of SNHL in the organ of Corti in vitro and establish a novel in vitro model for investigation of the mechanisms of SNHL.

\section{Data Availability}

The data that support the findings of this study are openly available from the corresponding author upon reasonable request.

\section{Conflicts of Interest}

The authors declare that there is no conflict of interests regarding the publication of this paper. 


\section{Acknowledgments}

This work was supported by grants from the National Natural Science Foundation of China (Grant Nos. 81830030, 81771016 , and 81700917). We would like to thank all the authors who contributed to this special issue.

\section{References}

[1] D. M. P. Jayakody, P. L. Friedland, R. N. Martins, and H. R. Sohrabi, "Impact of aging on the auditory system and related cognitive functions: a narrative review," Frontiers in Neuroscience, vol. 12, p. 125, 2018.

[2] S. Someya, T. Yamasoba, R. Weindruch, T. A. Prolla, and M. Tanokura, "Caloric restriction suppresses apoptotic cell death in the mammalian cochlea and leads to prevention of presbycusis," Neurobiology of Aging, vol. 28, no. 10, pp. 1613-1622, 2007.

[3] M. Knipper, P. Van Dijk, I. Nunes, L. Ruttiger, and U. Zimmermann, "Advances in the neurobiology of hearing disorders: recent developments regarding the basis of tinnitus and hyperacusis," Progress in Neurobiology, vol. 111, pp. 1733, 2013

[4] X. Fu, X. Sun, L. Zhang et al., "Tuberous sclerosis complexmediated mTORC1 overactivation promotes age-related hearing loss," The Journal of Clinical Investigation, vol. 128, no. 11, pp. 4938-4955, 2018.

[5] C. Cheng, Y. Wang, L. Guo et al., "Age-related transcriptome changes in Sox $2+$ supporting cells in the mouse cochlea," Stem Cell Research \& Therapy, vol. 10, no. 1, p. 365, 2019.

[6] A. R. Fetoni, F. Paciello, R. Rolesi, G. Paludetti, and D. Troiani, "Targeting dysregulation of redox homeostasis in noiseinduced hearing loss: oxidative stress and ROS signaling," Free Radical Biology \& Medicine, vol. 135, pp. 46-59, 2019.

[7] S. G. Kujawa and M. C. Liberman, "Adding insult to injury: cochlear nerve degeneration after "temporary" noise-induced hearing loss," The Journal of Neuroscience, vol. 29, no. 45, pp. 14077-14085, 2009.

[8] C. Fujimoto and T. Yamasoba, "Mitochondria-targeted antioxidants for treatment of hearing loss: a systematic review," Antioxidants, vol. 8, no. 4, p. 109, 2019.

[9] M. Jiang, T. Karasawa, and P. S. Steyger, "Aminoglycosideinduced cochleotoxicity: a review," Frontiers in Cellular Neuroscience, vol. 11, p. 308, 2017.

[10] Z. Zhong, X. Fu, H. Li et al., "Citicoline protects auditory hair cells against neomycin-induced damage," Frontiers in Cell and Development Biology, vol. 8, p. 712, 2020.

[11] S. Morioka, H. Sakaguchi, T. Yamaguchi et al., "Hearing vulnerability after noise exposure in a mouse model of reactive oxygen species overproduction," Journal of Neurochemistry, vol. 146, no. 4, pp. 459-473, 2018.

[12] R. S. Balaban, S. Nemoto, and T. Finkel, "Mitochondria, oxidants, and aging," Cell, vol. 120, no. 4, pp. 483-495, 2005.

[13] Y. Ohinata, J. M. Miller, and J. Schacht, "Protection from noise-induced lipid peroxidation and hair cell loss in the cochlea," Brain Research, vol. 966, no. 2, pp. 265-273, 2003.

[14] N. Benkafadar, F. Francois, C. Affortit et al., "ROS-induced activation of DNA damage responses drives senescence-like state in postmitotic cochlear cells: implication for hearing preservation," Molecular Neurobiology, vol. 56, no. 8, pp. 59505969, 2019.
[15] H. Yamane, Y. Nakai, M. Takayama, H. Iguchi, T. Nakagawa, and A. Kojima, "Appearance of free radicals in the guinea pig inner ear after noise-induced acoustic trauma," European Archives of Oto-Rhino-Laryngology, vol. 252, no. 8, pp. 504508, 1995.

[16] J. Menardo, Y. Tang, S. Ladrech et al., "Oxidative stress, inflammation, and autophagic stress as the key mechanisms of premature age-related hearing loss in SAMP8 mouse cochlea," Antioxidants \& Redox Signaling, vol. 16, no. 3, pp. 263-274, 2012.

[17] D. Mukherjea, S. Jajoo, T. Kaur, K. E. Sheehan, V. Ramkumar, and L. P. Rybak, "Transtympanic administration of short interfering (si)RNA for the NOX3 isoform of NADPH oxidase protects against cisplatin-induced hearing loss in the rat," Antioxidants \& Redox Signaling, vol. 13, no. 5, pp. 589-598, 2010.

[18] J. Guo, R. Chai, H. Li, and S. Sun, "Protection of hair cells from ototoxic drug-induced hearing loss," Advances in Experimental Medicine and Biology, vol. 1130, pp. 17-36, 2019.

[19] M. C. Liberman and S. G. Kujawa, "Cochlear synaptopathy in acquired sensorineural hearing loss: manifestations and mechanisms," Hearing Research, vol. 349, pp. 138-147, 2017.

[20] I. Varela-Nieto, S. Murillo-Cuesta, M. Calvino, R. Cediel, and L. Lassaletta, "Drug development for noise-induced hearing loss," Expert Opinion on Drug Discovery, vol. 15, no. 12, pp. 1457-1471, 2020.

[21] K. A. Fernandez, D. Guo, S. Micucci, V. De Gruttola, M. C. Liberman, and S. G. Kujawa, "Noise-induced cochlear synaptopathy with and without sensory cell loss," Neuroscience, vol. 427, pp. 43-57, 2020.

[22] H. Zhou, X. Qian, N. Xu et al., "Disruption of Atg7-dependent autophagy causes electromotility disturbances, outer hair cell loss, and deafness in mice," Cell Death \& Disease, vol. 11, no. 10, p. 913,2020

[23] Q. Jieyu, Z. Liyan, T. Fangzhi et al., "Espin distribution as revealed by super-resolution microscopy of stereocilia," American Journal of Translational Research, vol. 12, no. 1, 2020.

[24] D. Yamashita, H. Y. Jiang, J. Schacht, and J. M. Miller, "Delayed production of free radicals following noise exposure," Brain Research, vol. 1019, no. 1-2, pp. 201-209, 2004.

[25] K. Baker and H. Staecker, "Low dose oxidative stress induces mitochondrial damage in hair cells," The Anatomical Record, vol. 295, no. 11, pp. 1868-1876, 2012.

[26] K. Wakabayashi, M. Fujioka, S. Kanzaki et al., "Blockade of interleukin-6 signaling suppressed cochlear inflammatory response and improved hearing impairment in noisedamaged mice cochlea," Neuroscience Research, vol. 66, no. 4, pp. 345-352, 2010.

[27] S. Orrenius, B. Zhivotovsky, and P. Nicotera, "Regulation of cell death: the calcium-apoptosis link," Nature Reviews. Molecular Cell Biology, vol. 4, no. 7, pp. 552-565, 2003.

[28] B. H. Ahn, H. S. Kim, S. Song et al., "A role for the mitochondrial deacetylase Sirt3 in regulating energy homeostasis," Proceedings of the National Academy of Sciences of the United States of America, vol. 105, no. 38, pp. 1444714452, 2008.

[29] N. R. Sundaresan, M. Gupta, G. Kim, S. B. Rajamohan, A. Isbatan, and M. P. Gupta, "Sirt3 blocks the cardiac hypertrophic response by augmenting Foxo3a-dependent antioxidant defense mechanisms in mice," The Journal of Clinical Investigation, vol. 119, no. 9, pp. 2758-2771, 2009. 
[30] R. Tao, M. C. Coleman, J. D. Pennington et al., "Sirt3-mediated deacetylation of evolutionarily conserved lysine 122 regulates MnSOD activity in response to stress," Molecular Cell, vol. 40, no. 6, pp. 893-904, 2010.

[31] K. D. Brown, S. Maqsood, J. Y. Huang et al., "Activation of SIRT3 by the NAD ${ }^{+}$Precursor Nicotinamide Riboside Protects from Noise-Induced Hearing Loss," Cell Metabolism, vol. 20, no. 6, pp. 1059-1068, 2014.

[32] H. Pi, S. Xu, R. J. Reiter et al., "SIRT3-SOD2-mROS-dependent autophagy in cadmium-induced hepatotoxicity and salvage by melatonin," Autophagy, vol. 11, no. 7, pp. 1037-1051, 2015.

[33] U. Galli, O. Mesenzani, C. Coppo et al., "Identification of a sirtuin 3 inhibitor that displays selectivity over sirtuin 1 and 2," European Journal of Medicinal Chemistry, vol. 55, pp. 58-66, 2012.

[34] Y. Wang, J. Li, X. Yao et al., "Loss of CIB2 causes profound hearing loss and abolishes mechanoelectrical transduction in mice," Frontiers in Molecular Neuroscience, vol. 10, p. 401, 2017.

[35] Z. Shasha, Q. Ruiying, D. Ying et al., "Hair cell regeneration from inner ear progenitors in the mammalian cochlea," American Journal of Stem Cells, vol. 9, no. 3, 2020.

[36] R. Taylor, A. Bullen, S. L. Johnson et al., "Absence of plastin 1 causes abnormal maintenance of hair cell stereocilia and a moderate form of hearing loss in mice," Human Molecular Genetics, vol. 24, no. 1, pp. 37-49, 2015.

[37] Y. Sergeyenko, K. Lall, M. C. Liberman, and S. G. Kujawa, "Age-related cochlear synaptopathy: an early-onset contributor to auditory functional decline," The Journal of Neuroscience, vol. 33, no. 34, pp. 13686-13694, 2013.

[38] J. D. Goutman, A. B. Elgoyhen, and M. E. Gomez-Casati, "Cochlear hair cells: the sound-sensing machines," FEBS Letters, vol. 589, no. 22, pp. 3354-3361, 2015.

[39] A. C. Wong and A. F. Ryan, "Mechanisms of sensorineural cell damage, death and survival in the cochlea," Frontiers in Aging Neuroscience, vol. 7, p. 58, 2015.

[40] G. C. Kujoth, A. Hiona, T. D. Pugh et al., "Mitochondrial DNA mutations, oxidative stress, and apoptosis in mammalian aging," Science, vol. 309, no. 5733, pp. 481-484, 2005.

[41] B. Guo, Q. Guo, Z. Wang et al., "D-Galactose-induced oxidative stress and mitochondrial dysfunction in the cochlear basilar membrane: an In Vitro aging model," Biogerontology, vol. 21, no. 3, pp. 311-323, 2020.

[42] T. Kamogashira, C. Fujimoto, and T. Yamasoba, "Reactive oxygen species, apoptosis, and mitochondrial dysfunction in hearing loss," BioMed Research International, vol. 2015, Article ID 617207, 7 pages, 2015.

[43] S. W. Tait and D. R. Green, "Caspase-independent cell death: leaving the set without the final cut," Oncogene, vol. 27, no. 50, pp. 6452-6461, 2008.

[44] A. Kurabi, E. M. Keithley, G. D. Housley, A. F. Ryan, and A. C. Wong, "Cellular mechanisms of noise-induced hearing loss," Hearing Research, vol. 349, pp. 129-137, 2017.

[45] V. Warnsmann, N. Meyer, A. Hamann, D. Kogel, and H. D. Osiewacz, "A novel role of the mitochondrial permeability transition pore in (-)-gossypol-induced mitochondrial dysfunction," Mechanisms of Ageing and Development, vol. 170, pp. 45-58, 2018.

[46] T. N. Le, L. V. Straatman, J. Lea, and B. Westerberg, "Current insights in noise-induced hearing loss: a literature review of the underlying mechanism, pathophysiology, asymmetry, and management options," Journal of Otolaryngology - Head \& Neck Surgery, vol. 46, no. 1, p. 41, 2017.

[47] K. Hill, H. Yuan, X. Wang, and S. H. Sha, "Noise-induced loss of hair cells and cochlear synaptopathy are mediated by the activation of AMPK," The Journal of Neuroscience, vol. 36, no. 28, pp. 7497-7510, 2016.

[48] M. Kobel, C. G. Le Prell, J. Liu, J. W. Hawks, and J. Bao, "Noise-induced cochlear synaptopathy: past findings and future studies," Hearing Research, vol. 349, pp. 148-154, 2017.

[49] J. B. Jensen, A. C. Lysaght, M. C. Liberman, K. Qvortrup, and K. M. Stankovic, "Immediate and delayed cochlear neuropathy after noise exposure in pubescent mice," PLoS One, vol. 10, no. 5, article e0125160, 2015.

[50] L. A. Sena and N. S. Chandel, "Physiological roles of mitochondrial reactive oxygen species," Molecular Cell, vol. 48, no. 2, pp. 158-167, 2012.

[51] E. P. Yu and M. R. Bennett, "The role of mitochondrial DNA damage in the development of atherosclerosis," Free Radical Biology \& Medicine, vol. 100, pp. 223-230, 2016.

[52] Z. D. Du, S. Yu, Y. Qi et al., "NADPH oxidase inhibitor apocynin decreases mitochondrial dysfunction and apoptosis in the ventral cochlear nucleus of D-galactose-induced aging model in rats," Neurochemistry International, vol. 124, pp. 31-40, 2019.

[53] A. H. Tseng, S. S. Shieh, and D. L. Wang, "SIRT3 deacetylates FOXO3 to protect mitochondria against oxidative damage," Free Radical Biology \& Medicine, vol. 63, pp. 222-234, 2013.

[54] C. Han and S. Someya, "Maintaining good hearing: calorie restriction, Sirt3, and glutathione," Experimental Gerontology, vol. 48, no. 10, pp. 1091-1095, 2013.

[55] S. Someya, W. Yu, W. C. Hallows et al., "Sirt3 mediates reduction of oxidative damage and prevention of age-related hearing loss under caloric restriction," Cell, vol. 143 , no. 5, pp. $802-$ $812,2010$.

[56] B. Osborne, N. L. Bentley, M. K. Montgomery, and N. Turner, "The role of mitochondrial sirtuins in health and disease," Free Radical Biology \& Medicine, vol. 100, pp. 164-174, 2016.

[57] W. He, J. C. Newman, M. Z. Wang, L. Ho, and E. Verdin, "Mitochondrial sirtuins: regulators of protein acylation and metabolism," Trends in Endocrinology and Metabolism, vol. 23, no. 9, pp. 467-476, 2012.

[58] W. Yu, K. E. Dittenhafer-Reed, and J. M. Denu, "SIRT3 Protein Deacetylates Isocitrate Dehydrogenase 2 (IDH2) and Regulates Mitochondrial Redox Status," The Journal of Biological Chemistry, vol. 287, no. 17, pp. 14078-14086, 2012.

[59] M. Ristow and K. Zarse, "How increased oxidative stress promotes longevity and metabolic health: the concept of mitochondrial hormesis (mitohormesis)," Experimental Gerontology, vol. 45, no. 6, pp. 410-418, 2010.

[60] M. D. Hirschey, T. Shimazu, E. Jing et al., "SIRT3 deficiency and mitochondrial protein hyperacetylation accelerate the development of the metabolic syndrome," Molecular Cell, vol. 44, no. 2, pp. 177-190, 2011.

[61] D. A. Sinclair, "Toward a unified theory of caloric restriction and longevity regulation," Mechanisms of Ageing and Development, vol. 126, no. 9, pp. 987-1002, 2005.

[62] M. D. Hirschey, T. Shimazu, E. Goetzman et al., "SIRT3 regulates mitochondrial fatty-acid oxidation by reversible enzyme deacetylation," Nature, vol. 464, no. 7285, pp. 121$125,2010$. 
[63] P. I. Merksamer, Y. Liu, W. He, M. D. Hirschey, D. Chen, and E. Verdin, "The sirtuins, oxidative stress and aging: an emerging link," Aging (Albany NY), vol. 5, no. 3, pp. 144-150, 2013.

[64] O. Ozden, S. H. Park, H. S. Kim et al., "Acetylation of MnSOD directs enzymatic activity responding to cellular nutrient status or oxidative stress," Aging (Albany NY), vol. 3, no. 2, pp. 102-107, 2011.

[65] H. Kim, Y. D. Lee, H. J. Kim, Z. H. Lee, and H. H. Kim, “SOD2 and Sirt3 control osteoclastogenesis by regulating mitochondrial ROS," Journal of Bone and Mineral Research, vol. 32, no. 2, pp. 397-406, 2017.

[66] M. C. Chen, L. L. Hsu, S. F. Wang, C. Y. Hsu, H. C. Lee, and L. M. Tseng, "ROS mediate xCT-dependent cell death in human breast cancer cells under glucose deprivation," Cell, vol. 9, no. 7, p. 1598, 2020.

[67] Y. Quan, L. Xia, J. Shao et al., “Adjudin protects rodent cochlear hair cells against gentamicin ototoxicity via the SIRT3-ROS pathway," Scientific Reports, vol. 5, no. 1, p. 8181, 2015. 